\title{
Evolution of Gas-Filled Nanocracks in Crystalline Solids
}

\author{
M. Hartmann and H. Trinkaus \\ Institut für Festkörperforschung, Forschungszentrum Jülich, D-52425 Jülich, Germany
}

(Received 20 September 2001; published 18 January 2002)

\begin{abstract}
In this work, the evolution of gas-filled cracks under gas implantation and subsequent annealing is studied on the basis of an elastic continuum approach. The observed growth limitation of He-filled nanocracks in $\mathrm{SiC}$ is attributed to their stabilization by the formation of circular dislocation dipoles. The formation and Ostwald ripening of bubble-loop complexes at elevated temperatures is modeled in terms of gas atom exchange between such complexes coupled with local matrix atom exchange between bubbles and loops of the same complex. The scaling laws derived for the time dependence of bubble and loop sizes are found to be in good agreement with experimental data.
\end{abstract}

DOI: $10.1103 /$ PhysRevLett.88.055505

Solid systems far from equilibrium play a key role in modern material science and technology. A special class of such systems is formed by solids implanted with gas atoms of low solubility, particularly with inert gas atoms, which strongly tend to precipitate into bubbles [1]. The broad interest in this class of systems is due to their wide application ranging from microelectronic to nuclear materials.

Bubble formation in solids generally requires both gas and matrix atom diffusion. In a crystalline matrix, light gas atoms such as $\mathrm{H}$ and $\mathrm{He}$ commonly diffuse as interstitial atoms. There are medium temperatures where such interstitial gas atoms are already mobile while matrix atoms are still immobile. A process, for which no matrix atom transport is required, is the precipitation of the mobile gas atoms between atomic layers of the matrix lattice thus forming gas-filled Griffith cracks.

The present work on the evolution of gas-filled cracks was motivated by the observation of penny-shaped $\mathrm{nm}$-scale He platelets in $\mathrm{SiC}$ formed during He implantation at room temperature (RT) and subsequent thermal annealing [2,3]. Similar, though less pronounced, features have been observed before in some metals [4] and in $\mathrm{Si}$ [5]. Recent experimental studies indicate that the formation of gas-filled nanocracks is a general phenomenon in covalent (ceramic) materials $[3,6]$.

For the case of He-implanted $\mathrm{SiC}$, the following puzzling experimental observations have to be rationalized: (1) the uniform size of the observed nanoplatelets formed during He implantation at RT and its maintenance upon annealing up to about $1300 \mathrm{~K}$ without any indication of growth as expected for normal gas bubbles [7,8], and (2) at higher temperatures the formation and coarsening of complexes of bubbles and dislocation loops of self-interstitial type, in which both components occupy the same, even if growing, total volumes.

In the reports on these experimental features [2,3], some qualitative ideas for explaining them have been presented. (1) The growth limitation of $\mathrm{He}$ platelets has been attributed to their trapping by circular dislocation dipoles assumed to form close to their rim at some critical size, and
PACS numbers: 61.72.Bb, 61.72.Qq, 61.72.Yx, 62.20.Mk

to stay there at temperatures where both dislocation glide and climb are negligible. (2) The formation and coarsening of bubble-loop complexes at equal total volumes of the two components has been explained by a two-component Ostwald ripening process in which gas atoms are exchanged between different complexes by long range bulk diffusion while matrix atom diffusion is restricted to the surfaces of bubbles and to the cores of the dislocation loops, thus allowing only a local transfer of matrix atoms from bubbles to associated loops. In the present paper, we use analytical approximations to quantify these ideas and compare our results with the corresponding experimental results.

We first consider the postulated growth limitation of a gas-filled crack of radius $R_{c}$ caused by a circular dislocation dipole of radius $R_{l}$ forming at its periphery (Fig. 1). The formation of the loop dipole driven by the gas pressure inside the crack may be imagined to be the result of a separation of two loops of opposite Burgers vectors $\pm \underline{b}$ (and opposite types, with the one of vacancy type at the crack side and the one of self-interstitial type away from the crack) initially coinciding at $z=z_{0}$ and finally separated by $\Delta z=z_{1}-z_{0}$. (For simplicity, we assume $\underline{b}$ to be perpendicular to the crack plane). Since we want to treat the problem analytically, we describe the crystal lattice distorted around the crack and the loop dipole by an elastic continuum, and write the total free energy of the crack-loop configuration as

$$
F_{\mathrm{tot}}=2 \pi R_{c}^{2} \gamma+\frac{\pi^{2}}{3} \frac{\mu}{(1-\nu)} R_{c} u_{0}^{2}+F_{g}+F_{l}+W_{\mathrm{cl}},
$$

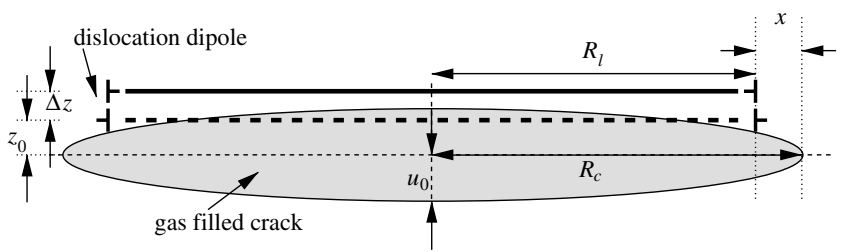

FIG. 1. Geometry of a gas-filled crack associated with dislocation dipole. 
where the first three terms are the contributions from the surface ( $\gamma$ : specific surface free energy), the elastic field ( $\mu$ : shear modulus, $\nu$ : Poisson's ratio, $u_{0}$ : crack opening displacement) and the gas of the original crack without loop dipole ("Griffith crack" [9]). The additional two terms are the contributions from the self-energy of the undisturbed loop dipole and its elastic interaction with the crack.

The self-energy of the loop dipole $F_{l}$ may be considered to be the energy required in the undisturbed lattice to separate two initially coinciding dislocation loops of radius $R_{l}$ and opposite Burgers vector $\pm \underline{b}$ by a spacing $\Delta z$. Using the linear elastic continuum approach, dislocation cores must be taken into account separately. Additionally, $F_{l}$ must be considered to be periodic in the discrete lattice with periodicity $b$ such that $\Delta z$ is a multiple of $b$ for equilibrium configurations. In covalent (unlike as for metallic) crystals, the barrier between two neighboring equilibrium configurations is that high that an increase in the loop separation (i.e., a glide of the upper loop in Fig. 1) even by only one Burgers vector requires high temperatures (above about $1 / 3$ of the melting temperature $T_{m}$ ) and/or a large shear stress driving the loop separation. Below $T_{m} / 3$, even the shear stress at the rim of the crack is able to separate the loops by only $\Delta z=b$. Since $F_{l}$ is irrelevant for the growth of the crack and its limitation at constant $R_{l}$, we do not specify any further details here.

On the other hand, the elastic interaction between the crack and a once formed loop dipole is crucial for the blocking of a further growth of the crack. A first order approximation for $W_{c l}$ in Eq. (1), in the spirit of perturbation approach, is obtained by integrating the forces, exerted by the stress field of the undisturbed crack (containing gas under constant pressure), on the two loops. Since both the postulated formation of the loop dipole and its blocking action on further crack growth will most favorably occur close to the periphery of the crack, the crack tip approximation for the stress field of the crack [10] may be used by which $W_{c l}$ can be written in the form

$$
\begin{gathered}
W_{c l}=\frac{\pi \mu b}{(1-\nu)} \sqrt{R_{c}} u_{0} \sqrt{|x|}\left[f\left(w_{1}\right)-f\left(w_{0}\right)\right], \\
f(w)=(w+\epsilon)^{3 / 2} / w, \\
w_{i}^{2}=1+\left(z_{i} / x\right)^{2}
\end{gathered}
$$

where $x=R_{c}-R_{l}$, and $\epsilon= \pm 1$ for $x \lessgtr 0$, respectively. In approximating the discrete lattice by an elastic continuum, the effective starting position for the loop separation $z_{0}$ remains uncertain within an atomic length scale. We expect that lattice effects can be simulated by choosing a proper value for $z_{0}$. Here, we have varied this quantity only from 0 to $b / 2$ and tested the sensitivity of our results with respect to this variation.

It is useful here to briefly discuss the gas-filled original crack without loop dipole ("Griffith crack" [9]), the free energy of which is described by the first three terms in Eq. (1). In this case, minimization of $F$ with respect to $u_{0}$ and $R_{c}$ yields the pressure $p_{c}$ and the displacement $u_{0}$ for a crack in equilibrium:

$$
\begin{gathered}
p_{c}=\frac{\pi \mu}{2(1-\nu)} \frac{u_{0}}{R_{c}}, \\
u_{0}^{2}=\frac{4(1-\nu) \gamma}{\pi \mu} R_{c} .
\end{gathered}
$$

For the observed cracklike He platelets in $\mathrm{SiC}$ with $u_{0} \approx$ $0.3 \mathrm{~nm}$ and $R_{c} \approx 4.5 \mathrm{~nm}$, Eq. (3a) yields, due to the high elastic strength of $\mathrm{SiC}(\mu=192 \mathrm{GPa}$ and $\nu=0.16)$, a remarkably high value for the pressure of about $24 \mathrm{GPa}$ where $\mathrm{He}$ is expected to be solid even up to $470 \mathrm{~K}$ [2].

An interesting quantity in the present context is the maximum shear stress component $\sigma_{x z}^{\max }$, driving loop dipole formation in the first atomic layer $\left(z_{0} \approx b / 2\right)$, which is given by

$$
\sigma_{x z}^{\max } \approx 0.55 \sqrt{\frac{\mu \gamma}{b(1-\nu)}} .
$$

For typical values of the material parameters $\gamma, \mu, \nu$, and $b$, Eq. (4) yields, independent of the crack size, values around or even above the theoretical shear strength $(\approx 0.2 \mu)$, indicating an intrinsic instability of gas-filled cracks in a crystalline solid to relax by dislocation dipole formation.

In fact, a necessary condition for the formation of a dislocation dipole at the rim of the crack is a decrease of the total free energy given by Eq. (1), i.e., that $W_{l}+W_{c l}<0$ for $\Delta z=b$. Additionally, a spontaneous formation of a dipole would require that $W_{l}+W_{c l}<0$ over the whole loop separation path $\Delta z$ which may be expected to be the case if the shear stress resolved along this path remains above the theoretical shear strength.

The equilibrium conditions for a crack in the presence of a once formed and then invariant loop dipole is obtained by minimization of the full free energy $F_{\text {tot }}$ given by Eq. (1) with respect to $u_{0}$ and $R_{c}$ at constant $R_{l}$. The resulting equilibrium pressure $p_{c l}$ exhibits a distinct deviation from the Griffith pressure $p_{c}$ around $R_{c} \approx R_{l}$, similar in shape to a resonance curve, as shown in Fig. 2. This feature is due to the passing of the crack tip from an energetically favorable region of dilatation to an unfavorable region of compression in the strain field of the loop dipole when $R_{c}$ passes $R_{l}$.

For crack growth, the functional dependencies of the pressure on crack size as shown in Fig. 2 have the following significance. Growth of an undisturbed Griffith crack by gas absorption from the environment results in a decrease of the pressure inside the crack and, according to the requirement of local chemical equilibrium, in a decrease of the local gas concentration in its environment. In a distribution of cracks of different sizes, the corresponding differences in the local gas concentrations lead to growth of larger cracks on the expense of smaller ones (Ostwald ripening).

The situation changes qualitatively upon the formation of a dislocation dipole by which the pressure, due to an 


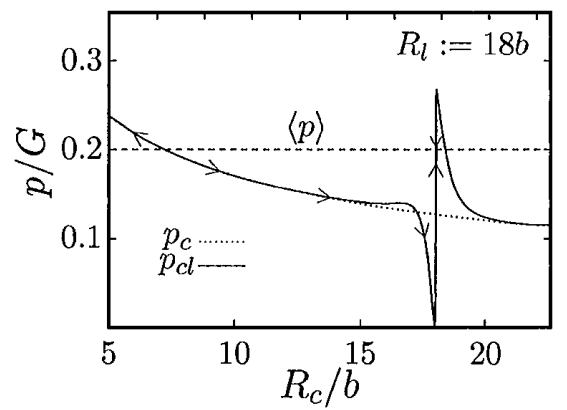

FIG. 2. Equilibrium gas pressure in a crack vs normalized crack radius without and with loop dipole (dotted and full lines) The dipole parameters were chosen to be $\Delta z=b$ and $z_{0}=0$. The radius of the loop dipole was assumed to be $18 b$ which corresponds to the radius of the He-platelets observed in $\mathrm{SiC}$ $[2,3]$. The horizontal line indicates schematically the average of the pressures in all cracks $\langle p\rangle$, assumed here to be dominated by the high pressures in small undisturbed Griffith cracks.

increase in the available volume, relaxes to a value close to the minimum in the corresponding curve in Fig. 2. Even a slight growth of such a crack results in a steep increase of the pressure inside and of the local gas concentration in its environment. The growth of the crack ceases completely when the local gas concentration in its environment has reached the average concentration defined by the average pressures in all cracks (with and without loop dipoles). In this situation, absorption and emission of gas atoms balance each other. Under continuous gas implantation or during annealing, small undisturbed Griffith cracks will grow until they reach the size where they can generate a dislocation dipole.

The necessity of a minimum crack size for dipole formation cannot be understood within the above used first order perturbation approximation where the reduction of the pressure upon dipole formation is neglected, which is justified only for sufficiently large cracks. In fact, the relative volume increase of the crack upon dipole formation $\Delta V / V$ decreases with increasing crack size since the volume gain, associated with the local dilatation due to the dipole, scales as $\Delta V \sim b^{2} R_{l}$, whereas the crack volume scales as $V \sim R_{c}^{2} u_{0}$, yielding $\Delta V / V \sim b^{2} / R_{c} u_{0}$ $\left(R_{l} \approx R_{c}\right)$. For too small cracks, on the other hand, the pressure decreases significantly along the path of the loop separation $\Delta z$, and exhausts before the dipole is completed. Therefore the critical size depends not only on the energetics of dipole formation but also on the equation of state of the gas. If the latter is available, the critical size can be estimated by assuming some plausible value for the maximum allowable relative pressure relaxation (say 25\%) [2]. A quantitative treatment in the framework of linear elastic continuum approach is not only very complicated but also doubtful.

Once formed crack-loop compounds of the shape described above stay in their metastable configuration as long as any form of matrix diffusion is negligible. At elevated temperature, where diffusion along surfaces and dislocation cores becomes significant but bulk diffusion is still negligible (in $\mathrm{SiC}$ at $1300 \mathrm{~K}<T<2200 \mathrm{~K}$ ), the low temperature configurations transform, under partial pressure relaxation, into energetically more favorable complexes consisting of now three-dimensional (spherical) bubbles and dislocation loops of self-interstitial type (the vacancy components of the dipoles are integrated into the bubbles), which subsequently begin to coarsen. The assumed restriction of matrix atom transport to internal surfaces and dislocation cores only is confirmed by the experimental observation that the total volumes of atoms of the bubble and the loop components are equal in such complexes [2,3]. According to this, the kinetic interaction between the complexes can be due only to $\mathrm{He}$ atom exchange between them.

Effects of kinetic restrictions on one of the components participating in bubble coarsening and their dependence upon the gas pressure in the bubbles have been discussed previously [11]. Unlike this we are confronted here with a completely new type of two-component Ostwald ripening process where the elements of one component, He atoms, are exchanged between the complexes by long ranging bulk diffusion while the diffusional transport of the other component, the matrix atoms, is restricted to the environment of each structure.

It is difficult to model the transformation from the low to high temperature configurations, particularly to different types of complexes with different degrees of complexity [3], where the initially high pressure is partially relaxed. In the following, we consider the coarsening of an existing system of bubble-loop complexes of the form illustrated in Fig. 3 (the simplest complex type observed [3]). The slower, and therefore rate controlling, of the two transfer processes is that of matrix atoms from the bubble to the associated loop, driven by differences in the chemical potential between both components. The chemical potentials of matrix atoms at a spherical bubble and a circular dislocation, respectively, are

$$
\begin{gathered}
\mu_{b}=\partial F_{b} / \partial N_{b} \quad=\left(p-2 \gamma / R_{b}\right) v, \\
\mu_{l}=\partial F_{l} / \partial N_{b} \quad=\tau v / b R_{l}=\tau v \kappa / b,
\end{gathered}
$$

where $N_{b}$ is the number of matrix atoms and $v$ their volume, $\tau \approx \mu b^{2}$ is the line tension of the dislocation and, $\kappa$ is the local curvature to be used for a noncircular

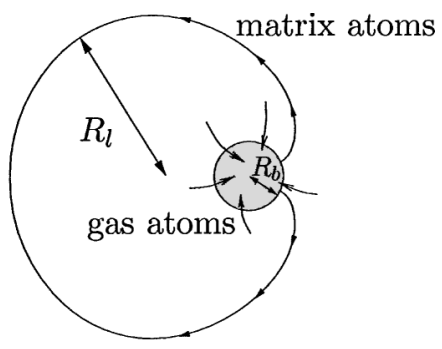

FIG. 3. Growth of a bubble-loop complex by the absorption of $\mathrm{He}$ atoms from the environment and the concurrent transfer of matrix atoms to the dislocation loop. 
dislocation as in the present case. At the entrance points from the bubble to the dislocation loop, $\kappa$ is determined by $\mu_{l}=\mu_{b}$. Matrix atoms flow from regions of large to those of small curvature. Thus, $\kappa$ decreases with increasing distance from the bubble for growing complexes, as shown in Fig. 3, and visa versa for shrinking complexes.

However, in the stage of coarsening, the change in $\kappa$ is that small that $\kappa \approx 1 / R_{l}$ may be used. Since the length scale over which the chemical potential changes is also $R_{l}$ (gradients $\sim 1 / R_{l}$ ), the flux of matrix atoms into the loop behaves as

$$
d N_{l} / d t \sim D_{d}\left(p-2 \gamma / R_{b}-\tau / b R_{l}\right) / R_{l},
$$

where $D_{d}$ is the coefficient of dislocation core diffusion. $R_{b}$ and $R_{l}$ are related to each other by the requirement of local matrix atom conservation

$$
4 \pi R_{b}^{3} / 3=\pi b R_{l}^{2} .
$$

According to Eq. (6), complex growth/shrinkage vanishes when

$$
p=2 \gamma / R_{b}^{*}-\tau / b R_{l}^{*} .
$$

For given $p$, corresponding to a given gas atom supersaturation, Eq. (8) defines the critical complex size $R_{b, l}^{*}$, below which the surface and line tensions press the gas out of the bubble, and above which the gas pumps up the bubble and the associated loop. Since gas atom exchange between complexes is much easier than matrix atom transfer from a bubble to an associated loop, the value of $p$ is approximately the same in all bubbles.

Since two differently growing spatial scales, $R_{b}$ and $R_{l}$, are contained in Eq. (6), the coarsening kinetics does not follow a unique temporal scaling law. However, at small and large complex sizes, i.e., in an early and a late stage, where the surface and line tension terms, respectively, are dominant in Eq. (6), the temporal evolution of the complexes can be well approximated by simple scaling laws. Using Eqs. (7) and (8) and ignoring in Eq. (6) the surface tension term in one and the line tension term in the other case, we obtain approximate scaling laws of the form

$$
R_{b} \sim\left(D_{d} t\right)^{x},
$$

with exponents $x=1 / 6 \approx 0.167$ and $x=2 / 11 \approx 0.182$ for the early and the late stage, respectively. Note that these values differ only slightly from each other but strongly from the value of $1 / 3$ for the normal coarsening of solid precipitates [12] and from the value of $1 / 2$ for the normal coarsening of ideal gas bubbles [7] or cracklike inclusions [13]. We have found that the experimentally observed more complicated bubble-loop complexes should coarsen with similar laws as the simple complexes considered here.

Finally, we compare our results with experimental data reported in Ref. [3]. The range of the exponent derived here, $0.167<x<0.182$, is in excellent agreement with the value of 0.17 obtained from fitting the respective experimental data. According to Eq. (9), the apparent activation energy of $R_{b}$ should be $x E_{d}$ where $E_{d}$ is the activation energy for dislocation core diffusion. Since the latter is expected to be around $1 / 2$ of the bulk self-diffusion energy of about $8 \mathrm{eV}$, we estimate $x E_{d} \approx 0.7 \mathrm{eV}$, in good agreement with the value of $0.75 \mathrm{eV}$ obtained from fitting the corresponding experimental data. We consider these agreements as strong evidence for the validity of our description of the coarsening of bubble-loop complexes.

In summary, we may say that we succeeded in explaining and describing the peculiarities in the evolution of gas platelets and bubbles in $\mathrm{SiC}$ in terms of two types of kinetic restrictions: (1) the blocking of platelet growth by the formation of immobile dislocation dipoles at low temperatures, and (2) the restriction of matrix atom transport away from a bubble surface to the core of an associated dislocation loop at elevated temperatures.

We conclude by emphasizing that modern material processing consists of the production of solid systems far from equilibrium and of the subsequent controlled release of some of their kinetic restrictions. An interesting example fitting into the present context is strain relaxation in $\mathrm{GeSi}$ on $\mathrm{Si}$ heterostructures by gas $(\mathrm{H}$ or $\mathrm{He}$ ) implantation and subsequent annealing where the dislocation loops formed at room temperature by cracklike cavities act at elevated temperature as dislocation sources required for the relaxation process [14].

We thank Professor Müller-Krumbhaar for discussions.

[1] For review, see Fundamental Aspects of Inert Gases in Solids, NATO ASI Ser. B, Vol. 279 (Plenum Press, New York, 1991).

[2] J. Chen, P. Jung, and H. Trinkaus, Phys. Rev. Lett. 82, 2709 (1999).

[3] J. Chen, P. Jung, and H. Trinkaus, Phys. Rev. B 61, 12923 (2000).

[4] T. Schober and H. Trinkaus, Philos. Mag. A 65, 1235 (1992).

[5] P. F. P. Fichtner, J. R. Kaschny, R. A. Yankov, A. Mückling, W. Kreisig, and W. Skorupa, Appl. Phys. Lett. 70, 732 (1997).

[6] J. Chen (private communications).

[7] A. J. Markworth, Metall. Trans. A 4, 2651-2656 (1973).

[8] H. Trinkaus, Radiat. Eff. 101, 91 (1986).

[9] I. N. Sneddon, Proc. R. Soc. London A 187, 229 (1946); see also T. Mura, Micromechanics of Defects in Solids (Nijhoff, The Hague, 1982).

[10] H.L. Ewalds and R.J.H. Wanhill, Fracture Mechanics (Arnold, London, 1984).

[11] H. Trinkaus, Scr. Metall. 23, 1773 (1989).

[12] I. M. Lifshitz and V. V. Slyozov, J. Chem. Solids 19, 35 (1961); C. Wagner, Z. Elektrochem. 65, 581 (1961).

[13] E. A. Brener, S. V. Iordanskii, and V. I. Marchenko, Phys. Rev. Lett. 82, 1506 (1999).

[14] H. Trinkaus, B. Holländer, St. Rongen, S. Mantl, H. J. Herzog, J. Kuchenbecker, and T. Hackbarth, App. Phys. Lett. 76, 3552 (2000). 